\title{
Coulisses
}

Revue de théâtre

\section{L'influence du théâtre japonais sur la pensée et le théâtre de Félix Guattari}

\section{Flore Garcin-Marrou}

\section{(2) OpenEdition}

1 Journals

Édition électronique

URL : https://journals.openedition.org/coulisses/745

DOI : $10.4000 /$ coulisses. 745

ISSN : 2546-9460

Éditeur

Presses universitaires de Franche-Comté

\section{Édition imprimée}

Date de publication : 31 décembre 2010

Pagination : 41-52

ISBN : 978-2-84867-302-8

ISSN : $1150-594 X$

\section{Référence électronique}

Flore Garcin-Marrou, «L'influence du théâtre japonais sur la pensée et le théâtre de Félix Guattari », Coulisses [En ligne], 41 | Automne 2010, mis en ligne le 30 novembre 2016, consulté le 30 décembre 2022. URL : http://journals.openedition.org/coulisses/745; DOI : https://doi.org/10.4000/coulisses 745 


\title{
L'influence du théâtre japonais sur la pensée et le théâtre de Félix Guattari
}

\author{
Flore Garcin-Marrou
}

1 Le philosophe français Félix Guattari (1930-1992) est surtout connu pour avoir écrit à quatre mains avec Gilles Deleuze les livres suivants : L'Anti-Oedipe (1972), Kafka. Pour une littérature mineure (1975), Rhizome (1976) repris dans Mille Plateaux (1980), et Qu'est-ce que la philosophie? (1991). En plus de son œuvre philosophique personnelle prolixe et complexe, Guattari s'est essayé à d'autres formes d'écritures de 1980 à 1990. Il a travaillé sur un roman autobiographique (33.333), sur des scénarios de films (Un Amour d'UIQ, Le Cahier vert), sur un recueil de poésie (Crac en plan pas un pli) et sur des pièces de théâtre. Il en existe six, inédites à ce jour et inconnues du public comme de la recherche universitaire. Retrouvées tout à fait par hasard il y a quelques mois dans les bibliothèques de certains de ses amis $^{1}$, ces pièces - je l'espère - sont destinées à vivre une deuxième jeunesse, en étant publiées très prochainement. Ma thèse en propose la première étude détaillée ${ }^{2}$.

2 L'Affaire du sac de chez Lancel, Le Maître de Lune, Psyche Ville Morte, Socrate, Visa le noir tua le blanc, et La Nuit la fin des moyens n'ont jamais été représentées sur scène. Seulement deux ont été mises en lecture : Socrate à Théâtre Ouvert à Paris en 1988, et La Nuit, la fin des moyens au Festival d'Avignon en $1990^{3}$. Ces pièces n'ont jamais rencontré leur public. Pourquoi ? D'un point de vue théâtral, elles présentent des difficultés certaines de mise en scène, dues à leurs espaces éclatés, aux chevauchements de temporalités, aux personnages désincarnés, aux situations indéfinies. Elles font partie de ce qu'Artaud nommait le «théâtre impossible ». C'est d'un point de vue philosophique qu'elles présentent plus d'intérêt, car elles sont un prolongement expérimental et artistique de la réflexion philosophique, une mise en exercice des concepts, une mise en présence scénique des idées. De quelle manière l'influence de l'Extrême-Orient et en particulier du Japon intervient-elle dans l'écriture de Félix Guattari ? Différentes formes de théâtre japonais ponctuent l'imaginaire du philosophe et participent activement à son univers de référence. La présence strictement citationnelle ou plus appuyée du kabuki, du 
bunraku et surtout de la danse des ténèbres, le butô, dans la réflexion et la dramaturgie du philosophe montre que sa pensée, puis son théâtre ont subi de façon effective l'influence du théâtre japonais. Qu'est-ce que Félix Guattari est allé chercher vers l'Orient, et surtout, qu'y a-t-il trouvé ?

Tout commence par la présence de deux élèves japonais à l'université de Vincennes dans les années 70 : Hidenobu Suzuki et Kuniichi Uno. Le premier est connu pour avoir enregistré les cours de Gilles Deleuze de 1979 à 1987 et le second pour avoir soutenu sa thèse sur Artaud $^{4}$ sous la direction de Deleuze en 1980 et avoir entretenu une correspondance avec son professeur ("Lettre à Uno sur le langage "; " Lettre à Uno : comment nous avons travaillé à deux $\left.{ }^{5} »\right)$. De retour au Japon, dès 1983, Kuniichi Uno se lance avec d'autres étudiants japonais dans la traduction de Mille Plateaux, encadré par le professeur Kouichi Toyosaki, traducteur de Rhizome, qui a été repris dans Mille Plateaux. Entre 1983 et 1990, Kuniichi Uno traduit L'Anti-Oedipe, L'Épuisé, Foucault, Le Pli, et devient professeur de littérature à l'université de Rykkio à Tokyo. La pensée de Deleuze et Guattari trouve un écho favorable au Japon, car c'est une pensée immanente refusant tout principe de transcendance, qui fait écho à la philosophie bouddhiste japonaise. En philosophie, est immanence ce qui est intérieur à l'être d'une réalité et ne renvoie, ni pour son existence, ni pour son explication, à aucun principe extérieur ou supérieur, c'est-à-dire à aucun principe transcendant. Le bouddhisme, lui, est perçu comme une forme de spiritualité sans dieu créateur, sans transcendance. Brahma n'est pas un être suprême, il se caractérise plutôt par son impersonnalité et son impermanence. La philosophie immanente ne rejette donc pas l'idée d'un dieu. Le dieu immanent se manifeste dans le monde, est présent dans chaque chose qui le compose, s'opposant à la conception d'un dieu transcendant, qui se situe en dehors, au-delà du monde.

4 Pour des raisons de santé, Deleuze ne fait jamais le voyage jusqu'au Japon malgré les nombreuses invitations de ses anciens élèves. Félix Guattari, lui, va s'y rendre une huitaine de fois en une décennie. Il est véritablement fasciné par le pays. En 1980 et en 1981, il se rend à Tokyo et enregistre des entretiens avec Tetsuo Kogawa ${ }^{6}$. En 1983, il revient sur l'invitation de Uno et d'Akira Asada de l'université de Kyoto, auteur la même année d'un best-seller sur la pensée française, La Structure et la force ${ }^{7}$. Le Japon déclenche en lui des réflexions, des fantasmes, des projets autour de sujets les plus divers, tels que la cuisine japonaise, l'architecture et les échanges artistiques interculturels entre la France et le Japon... C'est l'occasion pour Guattari de faire des conférences sur la schizoanalyse à l'université de Kyoto, de rencontrer des milieux politisés d'Okinawa, de visiter des hôpitaux psychiatriques en partageant son expérience de psychanalyste dans la clinique de psychiatrie alternative de La Borde, dans le Loir-et-Cher, fondée en 1953 par son ami Jean Oury8. La Borde vise depuis sa création à changer les méthodes de soin des schizophrènes, notamment en ne les enfermant plus, en les faisant participer aux tâches quotidiennes pour la bonne marche de l'institution ainsi qu'à de nombreux ateliers artistiques pour stimuler leur créativité. S'installe alors une réflexion commune entre la France et le Japon, qui perdure aujourd'hui, sur la psychothérapie institutionnelle, les bienfaits de l'art-thérapie, la lutte contre la passivité des patients et la nécessité de les inscrire dans un processus de création...

Dès son premier voyage en 1981, Félix Guattari assiste à des spectacles de bunraku et découvre cet art japonais $d u \mathrm{XVII}^{\mathrm{e}}$ siècle qui représente ses personnages par des 
marionnettes de grande taille, actionnées par trois manipulateurs se déplaçant en position de kathakali, jambes à demi-fléchies. Il y fait allusion lors d'un entretien avec Tetsuo Kogawa. La structure du bunraku lui permet d'étayer une métaphore de la famille française. Le journaliste japonais lui demande de la comparer à la famille japonaise. Guattari lui répond ceci: «En France, [...] rien n'a changé et même au contraire on est revenu en arrière. Il y a toute une mythologie, idéologie de la famille, de la femme au foyer, des enfants qui font très bien leurs études, [...]. C'est beaucoup lié au type de modèle familial véhiculé par les mass media, en particulier, par la télévision [...]. Vous savez, Freud, dans son étude sur le président Schreber dit que les personnes que le président Schreber voyait dans son délire étaient construites à la 6-4-2, un peu comme le théâtre bunraku. La famille française, c'est un peu une famille bunraku ; il y a la voix d'un côté, dans le poste de télévision, il y a les bras, les jambes qui partent un peu de tous les côtés, les équipements collectifs, les assistantes sociales qui tirent la mère d'un côté. C'est une famille totalement artificielle, ou je dirai, totalement déterritorialisée ». Mai 68 n'a visiblement pas fait évoluer la structure familiale française, qui reste sur le modèle oedipien triangulaire énoncé par Freud au xIX siècle. Un modèle qui vit son éclatement: la voix saisie par le discours des mass-media, les membres tiraillés par différentes institutions... « On fait tout pour que la marionnette familiale ressemble à une famille comme la marionnette bunraku! [...] On ne peut pas s'empêcher de penser que tout ça est complètement manipulé, articulé, par le pouvoir d'État, par le patronat, par la formation de la force collective de travail, par les équipements collectifs, par les mass media ! ». Guattari ici s'inspire de la particularité du bunraku (les marionnettes à manche manipulées par trois personnes) pour décrire la structure familiale telle qu'elle s'ordonne dans l'ordre capitaliste et médiatique. La famille est divisée, morcelée, afin que les formes de pouvoir aient un meilleur contrôle sur chaque partie de son corps. Cette réflexion fait référence aux thèses soutenues dans L'Anti-Oedipe, Capitalisme et schizophrénie 1, qui s'attache, dès 1972, comme l'indique d'emblée son titre, à contester le schéma oedipien hérité de Freud, censé régir chaque famille ou chaque micro-société. Pour Freud, la structure de la personnalité se construit d'après la seule triangulation oedipienne : chaque individu recherche le plaisir avec une personnalité maternelle et craint le père comme un rival et un castrateur potentiel, à l'image du personnage mythologique, héros des tragédies de Sophocle, Oedipe Roi et Oedipe à Colone. L'Anti-Oedipe propose une vision de la personnalité différente de l'interprétation oedipienne, considérée alors comme une véritable usine de production de flux de désir. Nous avons ici un premier exemple qui montre qu'une forme de théâtre japonais permet à Guattari de formuler une idée nouvelle. Le bunraku ayant ses propres codes, sa propre structure, Guattari déplace cette même structure dans un autre univers référentiel, l'univers familial. Le théâtre japonais ici, a un impact direct sur la pensée du philosophe ; il devient un outil philosophique.

6 Nous pouvons supposer que Félix Guattari a aussi assisté à une représentation de théâtre kabuki. Dans un texte inédit, "Tokyo l'orgueilleuse", il fait allusion à la posture des acteurs: " Les high ways, par trois étages de béton, qui enjambent la ville mosaïque, cuisses écartées à la façon des héros kabuki écrasant tout sur leur passage ${ }^{10}$ ». Cette référence picturale au kabuki fait référence au kata, qui est l'ensemble des gestes codifiés que les acteurs du kabuki doivent apprendre afin d'acquérir la souplesse et la puissance. Cette citation permet aussi d'évoquer la fascination de Félix Guattari pour l'architecture japonaise. Selon lui, ce pays a réussi la création singulière d'une subjectivité post-industrielle à travers la combinaison de l'ultra-moderne et de 
l'archaïque. Toute l'architecture est pensée d'après cette combinaison qui respecte la tradition, tout autant qu'elle innove. En 1987, la rencontre de Guattari avec l'architecte Shin Takamatsu, est animée par cette même problématique du croisement entre le modernisme technologique et l'archaïque ${ }^{11}$.

7 Après le bunraku et le kabuki, il s'agit de s'intéresser aux liens forts que Guattari tisse avec le danseur butô, Min Tanaka. Ce courant de danse des années 1950, créé par Tatsumi Hijikata (1928-1986) et Kazuo Ohno (né en 1906), cherche à recréer des nouvelles formes artistiques, tout en rendant hommage à la tradition. Sans s'encombrer du vocabulaire gestuel ancien du kabuki ou du nô, les danseurs cherchent à faire parler le corps plutôt qu'à créer un enchaînement de mouvements harmonieux. Cette réflexion sur le corps apparaît après le lancement de la bombe nucléaire sur Hiroshima : beaucoup d'artistes s'interrogent sur la représentation esthétique après la catastrophe et travaillent leur corps dans sa pure et stricte matérialité. Cette danse résonne en Europe chez les penseurs et artistes qui réfléchissent à l'après-Auschwitz ${ }^{12}$. Tatsumi Hijikata situe ses influences dans la littérature européenne: il cite Antonin Artaud, le marquis de Sade, Georges Bataille. Ce sont des références que Félix Guattari partage.

8 À la même époque, l'universitaire Georges Banu témoigne de l'engouement des intellectuels français pour Min Tanaka : «À Tôkyo, il y a plusieurs années, au plan B, seuls quelques étrangers éparpillés venaient voir danser Tanaka Min, tandis qu’à Paris au musée des Arts décoratifs, en 1978, dans le cadre du Festival d'Automne, c'était une foule qui se précipitait aux portes. De même qu'au Festival de Nancy en 1980, pour découvrir Kazuo Ohno ou Sankai Juku, tous deux révélés par Jean Kalman. Puis, invités dans la capitale, les danseurs du butô éclatèrent sur la scène occidentale ${ }^{13}$ \%. Selon Banu, le butô a rempli un horizon d'attente en Occident : il a montré la face cachée du Japon, la face immaîtrisable et hétérogène. Il combine tradition et modernité, Occident et Orient: «Si les formes anciennes - le nô, le bunraku, même le kabuki - se dressent comme des inaltérables rocs du passé, le butô apparaît telle une expression jaillie du

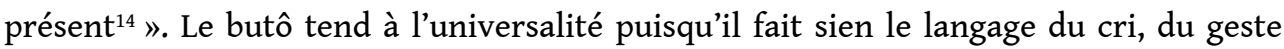
désordonné, non codifié : «On aime les haillons du butô en se rappelant la géométrie des costumes du kabuki, on se laisse emporter par la violence des spasmes en pensant à l'ordre secret qui régit les mouvements de l'acteur traditionnel, on est touché par l'eau, la terre et les saletés qui couvrent le plateau du butô en se souvenant de la scène glacée du nô où l'acteur se mire, on assiste à l'effort et à l'épuisement du danseur, tout en sachant que les acteurs de kabuki dissimulent la sueur sous le blanc du maquillage ${ }^{15}$ ». Enfin, Banu rejoint Guattari en concluant: "L'extrême contemporain et l'extrêmeancien ne font qu'un ${ }^{16} »$.

9 En 1984, Guattari écrit le texte de présentation du programme de danse butô de Min Tanaka, invité à Paris.

Tanaka Min

Le dauphin des ténèbres

Ses feux Zen sous les pas du miracle japonais

D'autres circonscriptions du sens

Un corps-sans-organe

En deça des identités industrielles

Au-delà des programmations narratives

Lenteurs à la vitesse de la lumière

Horizontalités animales

Pour arracher ses danses au cosmos

Coulisses, 41 | Automne 2010 
Diagrammes d'intensités

À l'intersection de toutes les scènes du possible

Chorégraphie d'un coup de dé du désir

Sur une ligne continue de naissance

Devenir irréversible des rythmes et ritournelles d'un événement-haïku

I dance not in the place but I dance the place

Tanaka Min

The body weather

Le roi nu de nos mémoires impossibles de l'être ${ }^{17}$.

10 Guattari constate qu'il y a de nombreux dénominateurs communs entre sa philosophie et le butô. L'immanence encore une fois en fait partie. La philosophie immanente évacue, nous l'avons vu, tout principe de transcendance. De même que la danse butô évacue les notions transcendantes de la danse occidentale qui vise par le contrôle, l'aisance et la beauté, à s'élever par la grâce toujours plus haut et au-delà... Le butô ne cherche pas à transcender le corps du danseur, mais à l'inscrire au contraire, dans l'immanence de la chair en lambeaux. Le butô donne à voir des corps réels. Le corps du danseur est, selon Hijikata : « Un cadavre qui ne tient debout qu'au péril de sa vie ». Ces corps intensifs ne sont autres que des "Corps sans organes" (noté à partir de maintenant, $\mathrm{CsO}$ ). Cette notion est empruntée à Antonin Artaud et largement développée dans le $6^{\mathrm{e}}$ plateau de Mille Plateaux, dont le titre est « 28 novembre 1947 Comment se faire un corps sans organes?». Le 28 Novembre 1947 correspond à l'écriture de la pièce radiophonique Pour en finir avec le Jugement de Dieu, date à laquelle Artaud déclare la guerre aux organes. Artaud pense que l'homme doit se faire « refaire son anatomie» :

[...] Lorsque vous lui aurez fait un corps sans organes,

alors vous l'aurez délivré de tous ses automatismes et rendu à sa véritable liberté.

Alors vous lui réapprendrez à danser à l'envers

comme dans le délire des bals musette

et cet envers sera son véritable endroit ${ }^{18}$.

Les organes symbolisent la structure, désignent un système qui organise des éléments entre eux. Or Artaud, comme Deleuze et Guattari s'opposent à l'idée de structure. Les deux philosophes se positionnent contre le structuralisme, courant de pensée qui leur est contemporain (dont les représentants sont Roland Barthes, Louis Althusser, Claude Lévi-Strauss...), en proposant une vision opposée, machinique et constructiviste de la vie. Alors que la structure coupe les flux, en les contraignant par une organisation arbitraire, le machinique permet la libre circulation des flux de désir : il permet à des agencements de se faire et de se défaire, sans jamais arrêter la chaîne de production. La réalité est un processus, et non pas un ensemble de signes. Elle est un work in progress, et non pas l'expression d'un Signifiant. L'homme troque son corps avec organe pour un $\mathrm{CsO}$ quand il ne supporte plus de se conformer à la structure et entend vivre d'après les intensités qui le traversent (ainsi parle-t-on du CsO du schizophrène, de l'anorexique, du toxicomane... mais aussi de l'artiste en création). Le CsO apparaît quand l'homme ne supporte plus « les yeux pour voir, les poumons pour respirer, la bouche pour avaler, la langue pour parler, le cerveau pour penser ». Voilà ce que veut traduire le danseur butô, ce danseur qui se donne pour mission de danser le cri du corps calciné de la victime d'Hiroshima, le cri de l'humanité à l'heure du post-nucléaire... Pour le danseur butô, la bombe a fait éclater toutes les structures, tous les signes et les Signifiants structurels : elle remet en cause le sens de l'existence. Deleuze et Guattari exhortent l'homme à trouver son $\mathrm{CsO}$, qui est un gage de liberté et d'émancipation : «Pourquoi 
pas marcher sur la tête, chanter avec les sinus, voir avec la peau, respirer avec le ventre $^{19}$ ? " La démarche du danseur butô Min Tanaka, dès 1979, va tout à fait dans ce même sens, lorsqu'il fonde le Body Weather Laboratory à Tokyo. Cette nouvelle approche, que l'on traduit par la «météorologie du corps » amène le danseur à travailler son corps, non pas comme une entité séparée de son esprit, mais dans son inter-relation avec l'environnement, la terre, les cultures, inspirée de la tradition Shintoïste. Rappelons qu'elle est la religion fondamentale la plus ancienne du Japon, dont le concept majeur est le caractère sacré de la nature. Le corps est relié aux forces de la nature. Le danseur butô travaille à fondre son corps dans le corps de la nature. Métaphoriquement, les organes du corps, qui sont des divisions de l'organisme, se diluent dans le Tout de la nature : ainsi naît le CsO du danseur butô.

Félix Guattari évoque également les vitesses, les flux d'intensités du butô qu'il assimile à des flux machiniques, qui se substituent aux signes du structuralisme : il ne s'agit plus de se demander ce que les choses signifient, mais comment les choses s'agencent entre elles. Deleuze et Guattari affirment que le monde peut être lu autrement que par la grille du signifiant (la grille de lecture qui pose la question: «Qu'est-ce que ça veut dire ?»). Il est aussi possible d'interpréter le monde selon les flux et les intensités qui le traverse, en se posant une autre question : celle du "Comment ça marche? ». "Au-delà des programmations narratives / Lenteurs à la vitesse de la lumière ${ }^{20} »$ est une sorte de haiku qui traduit le refus du signifiant, du narratif, au profit de l'émission des flux de désir. Les gestes du danseur butô ne racontent pas une histoire, mais doivent être ressentis comme des intensités émanant d'un «corps météorologique» («the body weather $\left.{ }^{21} »\right)$. Un corps qui est capable d'incarner non plus un personnage fixe, un rôle défini, mais plusieurs entités en devenir, dans une même performance, capable de capter l'atmosphère sèche, pluvieuse, caniculaire, neigeuse... Le corps peut renouer avec une certaine animalité, ces "Horizontalités animales", devenant fauve, félin, rampant... Cette capacité du danseur butô à embrasser différents devenirs est proche de ce que Deleuze et Guattari définissent comme «devenir-animal ». Développé dans Mille Plateaux ${ }^{22}$, le devenir-animal ne se " contente pas de passer par la ressemblance ${ }^{23}$ ", ni par l'imitation d'un animal : «Le devenir-animal de l'homme est réel, sans que soit réel l'animal qu'il devient ${ }^{24}{ }^{2}$. Il ne s'agit pas d'imiter un animal mais d'être pénétré par un devenir-animal : une rencontre entre deux règnes. Dans La Flûte enchantée, Mozart n'imite pas l'oiseau, mais sa musique est pénétrée par un devenir-oiseau ${ }^{25}$. Le danseur butô est à même d'être saisi par un devenir-imperceptible qui le traverse. Il n'est pas dans l'incarnation d'un personnage unique, mais se laisse traverser par des identités multiples, des trajectoires de vie.

Le danseur butô Kazuo Ohno ne cesse pas, depuis 1977, dans son Hommage à la Argentina de danser cette danseuse espagnole légendaire, qui a vécu au début du $\mathrm{xx}^{\mathrm{e}}$ siècle, surnommée la Pavlova du flamenco ${ }^{26}$. Pour cela, il ne l'imite pas mais est à l'écoute des «Diagrammes d'intensités / À l'intersection de toutes les scènes du possible / Chorégraphie d'un coup de dé du désir ${ }^{27} »$. Ces devenirs-imperceptibles, aussi présents dans le butô que dans la philosophie de Deleuze et Guattari, sont à l'image d'une nouvelle théorie de l'être, qui se définit non pas par une identité fixe, mais par sa capacité à être pris dans des devenirs : "Un corps ne se définit pas par la forme qui le détermine, ni comme une substance ou un sujet déterminés, ni par les organes qu'il possède ou les fonctions qu'il exerce. [...] Un corps se définit seulement par une longitude et une latitude: c'est-à-dire l'ensemble des éléments matériels qui lui 
appartiennent sous tels rapports de mouvement et de repos, de vitesse et de lenteur (longitude); l'ensemble des affects intensifs dont il est capable, sous tel pouvoir ou tel degré de puissance (latitude). Rien que des affects et des mouvements locaux, des vitesses différentielles ${ }^{28}$ ». Cette citation de Mille Plateaux rejoint le texte de Guattari sur Min Tanaka. Pour Félix Guattari, le butô est porteur d'une force révolutionnaire, participe à la constitution d'une nouvelle scène théâtrale, de nouveaux paradigmes esthétiques : «Le Butô est création continue et solitude. [...] Sans doute annonce-t-il l'entrée en scène de nouvelles façons d'être, moins fonctionnalistes, moins intellectualistes, moins détachées du corps et du Cosmos [...]. Oui, le Buto est une danse de lumière et de liberté29 ${ }^{2}$.

14 Si nous venons de voir de quelle manière le théâtre japonais avait influencé la pensée de Félix Guattari, nous pouvons dès lors poser quelques jalons pour l'analyse de son théâtre, peu connu, jamais étudié. Le théâtre d'Extrême-Orient a-t-il eu un impact sur ce que l'on pourrait nommer la dramaturgie guattarienne? Deux pièces sur six font référence à la culture chinoise et japonaise. Tout d'abord, Le Maitre de Lune, daté des années 1984-1985, met en scène trois groupes de trois acteurs, qui «animent trois grandes-marionnettes-totem d'inspiration kabuki ». Les manipulateurs sont notés pour la marionnette A: A1, A2, A3, pour la marionnette B : B1, B2, B3... Cet emprunt au kabuki vise à matérialiser scéniquement deux concepts chers à la philosophie de Guattari : l' "agencement collectif d'énonciation » et la "polyphonie ». L'orientalisme n'est pas décoratif, mais structure l'identité des personnages A, B, C. Le kabuki permet à Guattari d'expérimenter une nouvelle conception du personnage. La marionnette $\mathrm{A}$ s'exprime à travers trois voix :

A1 : Dès que je l'ai vue, un rêve d'iguane s'est posé sur ma nuque.

A2 : Ce n'était qu'une ombre portée sur le néant !

A3 : Une étreinte en creux, une sorte de $\operatorname{truc}^{30} \ldots$

15 Cette polyphonie traduit l'idée d'agencement collectif d'énonciation, qui correspond à une nouvelle vision de l'être, que nous avons abordée au sujet de la météorologie des corps de Min Tanaka. Le sujet n'est pas un, mais plusieurs. Il n'est pas identité, mais agencement collectif. Tout personnage réunit en lui-même différentes voix. Il en va de même pour le personnage de théâtre, qui ne se définit pas par une identité fixe, dont on pourrait dresser une histoire linéaire en lui attribuant une biographie imaginaire (c'est ce que recommande Constantin Stanislavski dans La Formation de l'acteur). Le personnage de théâtre n'est pas homogène, mais hétérogène. Il parle d'une voix polyphonique. Il se déplace selon des vitesses, des flux, des variations et n'est jamais mu par des motivations psychologiques : le personnage de théâtre capte les forces qui animent l'espace scénique. Le personnage de théatre incarne un $\mathrm{CsO}$, en devenir, vivant selon une formation d'un circuit d'intensités entre l'énergie féminine et l'énergie masculine (Yin et Yang). L'acteur chez Félix Guattari travaille comme le danseur butô.

La deuxième pièce qui fait référence à l'Extrême-Orient, La Nuit, la fin des moyens, a été écrite en 1990. Elle montre que les références à l'Orient peuvent être approximatives et fantasmatiques Son personnage principal féminin a un patronyme japonais: elle répond au nom de Midori. Mais, il semble qu'elle n'ait de japonais que le nom. Elle cite un des chefs d'œuvre de la littérature romanesque chinoise écrite au XIve siècle : «Il reprendra sa lecture du Shui-hu-zhuan, matrice surgelée aux épices vénéneuses, alors qu'il lui faudrait une surabondance d'être, une plénitude d'intensité qui le feraient dériver vers d'autres univers ». La référence n'a ici qu'une valeur citationnelle, qui n'a 
aucune incidence sur le cours de l'action. Plus loin, Midori donne son avis sur l'amour, évoquant le Yin et le Yang:

Midori : [...] À la différence des autres, je n'entretiens pas avec mes soi-disant semblables des rapports de complémentarité automatiques. Je ne marche pas dans leurs histoires de Yin et de Yang. Pour appréhender si peu que ce soit mon altérité, il est nécessaire de faire l'effort de sortir de soi-même et de se construire une extériorité, fusse de toutes pièces. Et d'ailleurs, on n'y parvient jamais

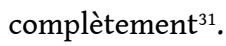

17 Elle continue en citant un long extrait d'un texte mystique de sainte Thérèse de Jésus. Ces discours de sources multiples, en apparence antagonistes, montrent que Midori est, comme la plupart des personnages de Guattari, une sorte d'antenne de radio (ou une station météorologique) qui capte les différentes ondes et flux qui traversent l'atmosphère. De cette manière, la cohérence du discours est bousculée. Les propos ne s'enchaînent pas comme le prescrit l'ordre du discours tel que l'usage commun le conçoit, mais ils s'enchaînent plutôt en suivant la mécanique du rêve, par association libre, de façon tout à fait aléatoire (Guattari évoque une «chorégraphie d'un coup de dé »), par superposition de strates d'univers multiréférentiels, qui place les références à l'Extrême-Orient au milieu de mille autres références.

C'est un constat de fait, le théâtre d'Extrême-Orient a eu une influence beaucoup plus importante sur la pensée de Guattari, que sur son théâtre. Deleuze et Guattari sont fortement marqués par la pensée d'Artaud. Ils ont une connaissance approfondie de son texte "Théâtre oriental et théâtre occidental ${ }^{32}$ ». Artaud a ouvert une fenêtre vers l'Orient : les deux philosophes se sont engouffrés dans cette lignée, mais en déplaçant la réflexion d'Artaud sur le théâtre, dans le domaine de la philosophie. De sorte que le théâtre d'Extrême-Orient ne leur sert pas à révolutionner les codes du théâtre occidental (mis à part la conception moderne du personnage comme agencement polyphonique), mais à révolutionner les codes de la philosophie contemporaine. De cette attirance vers l'ailleurs, découlent les concepts emblématiques de leur philosophie. La « déterritorialisation » consiste à rompre le lien de territorialité avec un territoire et une culture, pour tendre vers un autre territoire. Le "nomadisme » invite à adopter une optique de décentrement par rapport à la pensée occidentale. Le « $\mathrm{CsO}$ » est un corps oriental : le sinologue François Jullien le décrit non pas comme une charpente anatomique mais pensé « en mouvement selon un jeu complexe de circuits énergétiques, [...] en intime relation avec le monde ambiant : car tout le paysage, de même que le corps humain, est parcouru de souffles qui le font vibrer ${ }^{33}$ ». La découverte des pièces de théâtre de Félix Guattari va peut-être pouvoir permettre de rebasculer ces concepts philosophiques au sein d'une réflexion théâtrale et d'expérimenter quel pourrait être leur impact sur une pratique théâtrale : comment un acteur travaille-t-il avec l'idée de polyphonie, d'agencement collectif d'énonciation, de déterritorialisation, de nomadisme, de CsO ? 


\section{BIBLIOGRAPHIE}

BANU Georges, « Le Butô ou la subversion », L'Acteur qui ne revient pas. Journées du théâtre au Japon, Paris, Gallimard, 1993.

GARCIN-MARROU Flore, Gilles Deleuze, Félix Guattari : entre théâtre et philosophie, Thèse de Littérature française, Université Paris-IV Sorbonne.

GENOSKO Gary, Félix Guattari : an aberrant introduction, éd. NY Continuum, 2002, pp 128-129.

GUATTARI Félix, archives IMEC, Fonds Félix Guattari : ET02-12 « Tokyo l'orgueilleuse »; ET04-14

« Osaka»; ET04-23 «Proposition relative à l'organisation de manifestations d'artistes japonais ».

GUATTARI Félix, Théâtre complet, documentation personnelle, @ Enfants Guattari.

\section{NOTES}

1. Notamment Enzo Cormann, qui est le seul à avoir évoqué l'existence de ces documents dans son article "Comme sans y penser ", in Revue Chimères $n^{\circ} 23$ :

http://www.revue-chimeres.fr/drupal_chimeres/files/23chi02.pdf

2. Flore Garcin-Marrou, «Gilles Deleuze, Félix Guattari : entre théâtre et philosophie », Paris IVSorbonne.

3. La lecture de Socrate a eu lieu le 18 janvier 1988, dans le cadre d'une carte blanche à Enzo Cormann, sur l'invitation de Lucien Attoun. Le programme titre: "Socrate. Péripétie schizoanalytique : première ébauche ». Les comédiens sont Arnaud Carbonnier et Enzo Cormann. La lecture de La Nuit, la fin des moyens a eu lieu au gymnase Aubanel en Avignon, par la troupe des A.P. A (Artistes Producteurs Associés), sur l'initiative de Jean-Pierre Thibaudat.

4. Kuniichi Uno, Artaud et l'espace des forces, thèse de philosophie sous la direction de Gilles Deleuze, Université Paris 8, 1980.

5. Gilles Deleuze, Deux Régimes de fous, Paris, Éditions de Minuit, 2003, p. 185, p. 218

6. Les enregistrements des trois interviews de Félix Guattari avec Tetsuo Kogawa sont disponibles sur ce site : http://anarchy.translocal.jp/guattari/index.html

7. Akira Asada, Structure and Power, beyond semiotics, Tokyo, Keisō Shobō, 1983.

8. Le docteur Jean Oury a fait lui-même plusieurs fois le voyage jusqu'au Japon, afin de rencontrer les Associations d'art-thérapie et de parler sur « la psychothérapie institutionnelle et les activités d'expression artistique » : http://www.psychanalyse-en-mouvement.net/actualites/ article-79-2007050679-jean-oury-rencontre-avec-le-japon.html

9. Retranscription d'un entretien de Félix Guattari à Tokyo, avec Tetsuo Kogawa, sur la question de la famille. 22-05-1981, « Family Problems », http://anarchy.translocal.jp/guattari/index.html 10. Félix Guattari, « Tokyo l'orgueilleuse », Fonds Félix Guattari, IMEC.

11. Félix Guattari, «Les Machines architecturales de Shin Takamatsu », Revue Chimères, avec l'introduction de Christian Girard, «L'Architecture comme production de subjectivités », http:// www.revue-chimeres.fr/drupal_chimeres/files/21chi14.pdf Cf. aussi l'article de Gary Genosko, "An Introduction to Singularization and Style: Shin Takamatsu in Conversation with Félix Guattari », Parallax, Volume 7, Issue 4, Routledge, October 2001, p. 128-130.

12. Adorno est un des premiers à prévenir les écrivains qu'ils ne sauraient plus s'adonner sans péril à leur art: "Après Auschwitz, écrire un poème est barbare " (in Prismes et Dialectique négative). Pourtant certains témoignent avec difficulté de l'horreur après Auschwitz: Primo Levi 
dans La Trêve, Si c'est un homme, Jorge Semprun dans L'Ecriture ou la vie. Plus tard, Giorgio Agamben, dans Ce qui reste d'Auschwitz.

13. Georges Banu, "Le butô ou la subversion ", L'acteur qui ne revient pas. Journées du théâtre au Japon, Paris, Gallimard, 1993, p. 192-196.

14. Id., p. 195.

15. Id., p. 195.

16. Id., p. 196.

17. Félix Guattari, archives IMEC, Écrits théoriques, GTR2. Aa-15.44. Sous-titre : « Tanaka Min / le dauphin des ténèbres... » dactylographié, 1984.

18. Antonin Artaud, Oeuvres, «Pour en finir avec le jugement de dieu », Paris, Gallimard, Quarto, 2004, p. 1654.

19. Gilles Deleuze, Félix Guattari, Mille Plateaux, Paris, Éditions de Minuit, 1980, p. 187.

20. Félix Guattari, archives IMEC, Écrits théoriques, GTR2. Aa-15.44. Sous-titre : " Tanaka Min / le dauphin des ténèbres... » dactylographié, 1984.

21. Félix Guattari, « Tokyo l'orgueilleuse », Fonds Félix Guattari, IMEC.

22. Gilles Deleuze, Félix Guattari, Mille Plateaux, Paris, Éditions de Minuit, 1980, p. 284-381.

23. Id., p. 285.

24. Id., p. 291.

25. Le devenir-oiseau se lit dans le thème de l'oiseleur interprété par Papageno.

26. De cette étonnante performance de la Argentina par Kazuo Ohno, il existe quelques rares images : http://www.youtube.com/watch?v=2gqukIxf8oM

27. Félix Guattari, archives IMEC, Écrits théoriques, GTR2. Aa-15.44. Sous-titre : « Tanaka Min / le dauphin des ténèbres... » dactylographié 1984.

28. Gilles Deleuze, Félix Guattari, Mille Plateaux, Paris, Éditions de Minuit, 1980, p. 318.

29. Félix Guattari, Écrits théoriques, GTR2.Aa-15-51, Fonds Félix Guattari, IMEC.

30. Félix Guattari, Le Maître de Lune, documentation personnelle, @ Enfants Guattari, p. 28.

31. Félix Guattari, La Nuit, la fin des moyens, documentation personnelle, ( E Enfants Guattari, p. 28.

32. "La révélation du Théâtre Balinais a été de nous fournir du théâtre une idée physique et non verbale, où le théatre est contenu dans les limites de tout ce qui peut se passer sur une scène, indépendamment du texte écrit, au lieu que le théâtre tel que nous le concevons en Occident a partie liée avec le texte et se trouve limité par lui. », Antonin Artaud, Oeuvres, « Le Théâtre et son double ", Paris, Gallimard, Quarto, 2004, p. 545-549.

33. François Jullien, De l'Essence ou du Nu, Paris, Le Seuil, 2000, p. 74, p. 76.

\section{INDEX}

Palavras-chave : teatro japonês, pensamento, butô

Keywords : japanese theater, thought, butô

oeuvrecitee Anti-@idipe (L')

Palabras claves : teatro japonés, pensamiento, butô

Mots-clés : théâtre japonais, pensée, butô 
AUTEURS

FLORE GARCIN-MARROU

Paris IV - Sorbonne 